\title{
A new determinant expression of the zeta function for a hypergraph
}

\author{
Iwao Sato \\ Oyama National College of Technology, \\ Oyama, Tochigi 323-0806, Japan \\ isato@oyama-ct.ac.jp
}

Submitted: Mar 1, 2009; Accepted: Oct 19, 2009; Published: Oct 31, 2009

Mathematical Subject Classification: 05C50, 15A15

\begin{abstract}
Recently, Storm [10] defined the Ihara-Selberg zeta function of a hypergraph, and gave two determinant expressions of it by the Perron-Frobenius operator of a digraph and a deformation of the usual Laplacian of a graph. We present a new determinant expression for the Ihara-Selberg zeta function of a hypergraph, and give a linear algebraic proof of Storm's Theorem. Furthermore, we generalize these results to the Bartholdi zeta function of a hypergraph.
\end{abstract}

\section{Introduction}

Graphs and digraphs treated here are finite. Let $G$ be a connected graph and $D$ the symmetric digraph corresponding to $G$. Set $D(G)=\{(u, v),(v, u) \mid u v \in E(G)\}$. For $e=(u, v) \in D(G)$, set $u=o(e)$ and $v=t(e)$. Furthermore, let $e^{-1}=(v, u)$ be the inverse of $e=(u, v)$.

A path $P$ of length $n$ in $G$ is a sequence $P=\left(e_{1}, \cdots, e_{n}\right)$ of $n$ arcs such that $e_{i} \in$ $D(G), t\left(e_{i}\right)=o\left(e_{i+1}\right)(1 \leqslant i \leqslant n-1)$. If $e_{i}=\left(v_{i-1}, v_{i}\right)$ for $i=1, \cdots, n$, then we write $P=\left(v_{0}, v_{1}, \cdots, v_{n-1}, v_{n}\right)$. Set $|P|=n, o(P)=o\left(e_{1}\right)$ and $t(P)=t\left(e_{n}\right)$. Also, $P$ is called an $(o(P), t(P))$-path. We say that a path $P=\left(e_{1}, \cdots, e_{n}\right)$ has a backtracking or a bump at $t\left(e_{i}\right)$ if $e_{i+1}^{-1}=e_{i}$ for some $i(1 \leqslant i \leqslant n-1)$. A $(v, w)$-path is called a $v$-cycle (or $v$-closed path) if $v=w$. The inverse path of a path $P=\left(e_{1}, \cdots, e_{n}\right)$ is the path $P^{-1}=\left(e_{n}^{-1}, \cdots, e_{1}^{-1}\right)$.

We introduce an equivalence relation between cycles. Two cycles $C_{1}=\left(e_{1}, \cdots, e_{m}\right)$ and $C_{2}=\left(f_{1}, \cdots, f_{m}\right)$ are called equivalent if $f_{j}=e_{j+k}$ for all $j$. The inverse cycle of $C$ is not equivalent to $C$. Let $[C]$ be the equivalence class which contains a cycle $C$. Let $B^{r}$ be the cycle obtained by going $r$ times around a cycle $B$. Such a cycle is called a multiple of $B$. A cycle $C$ is reduced if both $C$ and $C^{2}$ have no backtracking. Furthermore, a cycle 
$C$ is prime if it is not a multiple of a strictly smaller cycle. Note that each equivalence class of prime, reduced cycles of a graph $G$ corresponds to a unique conjugacy class of the fundamental group $\pi_{1}(G, v)$ of $G$ at a vertex $v$ of $G$.

The Ihara-Selberg zeta function of $G$ is defined by

$$
\mathbf{Z}(G, t)=\prod_{[C]}\left(1-t^{|C|}\right)^{-1},
$$

where $[C]$ runs over all equivalence classes of prime, reduced cycles of $G$. Ihara [6] defined zeta functions of graphs, and showed that the reciprocals of zeta functions of regular graphs are explicit polynomials. A zeta function of a regular graph $G$ associated with a unitary representation of the fundamental group of $G$ was developed by Sunada [11,12]. Hashimoto [4] treated multivariable zeta functions of bipartite graphs. Bass [2] generalized Ihara's result on the zeta function of a regular graph to an irregular graph $G$.

Let $G$ be a connected graph with $n$ vertices and $m$ edges. Then two $2 m \times 2 m$ matrices $\mathbf{B}=\mathbf{B}(G)=\left(\mathbf{B}_{e, f}\right)_{e, f \in D(G)}$ and $\mathbf{J}_{0}=\mathbf{J}_{0}(G)=\left(\mathbf{J}_{e, f}\right)_{e, f \in D(G)}$ are defined as follows:

$$
\mathbf{B}_{e, f}=\left\{\begin{array}{ll}
1 & \text { if } t(e)=o(f), \\
0 & \text { otherwise }
\end{array}, \mathbf{J}_{e, f}= \begin{cases}1 & \text { if } f=e^{-1} \\
0 & \text { otherwise }\end{cases}\right.
$$

Theorem 1 (Bass) Let $G$ be a connected graph with $n$ vertices and $m$ edges. Then the reciprocal of the Ihara-Selberg zeta function of $G$ is given by

$$
\mathbf{Z}(G, t)^{-1}=\operatorname{det}\left(\mathbf{I}_{2 m}-t\left(\mathbf{B}-\mathbf{J}_{0}\right)\right)=\left(1-t^{2}\right)^{m-n} \operatorname{det}\left(\mathbf{I}_{n}-t \mathbf{A}(G)+t^{2}\left(\mathbf{D}_{G}-\mathbf{I}_{n}\right)\right),
$$

where $\mathbf{D}_{G}=\left(d_{i j}\right)$ is the diagonal matrix with $d_{i i}=\operatorname{deg}_{G} v_{i}\left(V(G)=\left\{v_{1}, \cdots, v_{n}\right\}\right)$.

The first identity in Theorem 1 was also obtained by Hashimoto [5]. Bass proved the second identity by using a linear algebraic method.

Stark and Terras [9] gave an elementary proof of this formula, and discussed three different zeta functions of any graph. Various proofs of Bass' Theorem were given by Kotani and Sunada [7], and Foata and Zeilberger [3].

Let $G$ be a connected graph. Then the cyclic bump count $\operatorname{cbc}(\pi)$ of a cycle $\pi=$ $\left(\pi_{1}, \cdots, \pi_{n}\right)$ is

$$
c b c(\pi)=\left|\left\{i=1, \cdots, n \mid \pi_{i}=\pi_{i+1}^{-1}\right\}\right|,
$$

where $\pi_{n+1}=\pi_{1}$.

Bartholdi [1] introduced the Bartholdi zeta function of a graph. The Bartholdi zeta function of $G$ is defined by

$$
\zeta(G, u, t)=\prod_{[C]}\left(1-u^{c b c(C)} t^{|C|}\right)^{-1}
$$

where $[C]$ runs over all equivalence classes of prime cycles of $G$, and $u, t$ are complex variables with $|u|,|t|$ sufficiently small.

Bartholdi [1] gave a determinant expression of the Bartholdi zeta function of a graph. 
Theorem 2 (Bartholdi) Let $G$ be a connected graph with $n$ vertices and $m$ unoriented edges. Then the reciprocal of the Bartholdi zeta function of $G$ is given by

$$
\begin{gathered}
\zeta(G, u, t)^{-1}=\operatorname{det}\left(\mathbf{I}_{2 m}-t\left(\mathbf{B}-(1-u) \mathbf{J}_{0}\right)\right) \\
=\left(1-(1-u)^{2} t^{2}\right)^{m-n} \operatorname{det}\left(\mathbf{I}-t \mathbf{A}(G)+(1-u)\left(\mathbf{D}_{G}-(1-u) \mathbf{I}\right) t^{2}\right) .
\end{gathered}
$$

Storm [10] defined the Ihara-Selberg zeta function of a hypergraph. A hypergraph $H=(V(H), E(H))$ is a pair of a set of hypervertices $V(H)$ and a set of hyperedges $E(H)$, which the union of all hyperedges is $V(H)$. In general, the union of all hyperedges is a subset of $V(H)$. For example, if a graph (that is, a 2-uniform hypergraph) has an isolated vertex, then the union of all edges is a proper subset of $V(H)$. A hypervertex $v$ is incident to a hyperedge $e$ if $v \in e$.

A bipartite graph $B_{H}$ associated with a hypergraph $H$ is defined as follows: $V\left(B_{H}\right)=$ $V(H) \cup E(H)$ and $v \in V(H)$ and $e \in E(H)$ are adjacent in $B_{H}$ if $v$ is incident to $e$. Let $V(H)=\left\{v_{1}, \ldots, v_{n}\right\}$. Then an adjacency matrix $\mathbf{A}(H)$ of $H$ is defined as a matrix whose rows and columns are parameterized by $V(H)$, and $(i, j)$-entry is the number of directed paths in $B_{H}$ from $v_{i}$ to $v_{j}$ of length 2 with no backtracking.

For the bipartite graph $B_{H}$ associated with a hypergraph $H$, let $V_{1}=V(H)$ and $V_{2}=E(H)$. Then, the halved graph $B_{H}^{[i]}$ of $B_{H}$ is defined to be the graph with vertex set $V_{i}$ and arc set $\left\{P\right.$ : reduced path ||$\left.P \mid=2 ; o(P), t(P) \in V_{i}\right\}$ for $i=1,2$.

Let $H$ be a hypergraph. A path $P$ of length $n$ in $H$ is a sequence $P=\left(v_{1}, e_{1}, v_{2}, e_{2}, \cdots\right.$, $\left.e_{n}, v_{n+1}\right)$ of $n+1$ hypervertices and $n$ hyperedges such that $v_{i} \in V(H), e_{j} \in E(H), v_{1} \in e_{1}$, $v_{n+1} \in e_{n}$ and $v_{i} \in e_{i}, e_{i-1}$ for $i=2, \ldots, n-1$. Set $|P|=n, o(P)=v_{1}$ and $t(P)=v_{n+1}$. Also, $P$ is called an $(o(P), t(P))$-path. We say that a path $P$ has a hyperedge backtracking if there is a subsequence of $P$ of the form $(e, v, e)$, where $e \in E(H), v \in V(H)$. A $(v, w)$-path is called a $v$-cycle (or $v$-closed path) if $v=w$.

We introduce an equivalence relation between cycles. Two cycles $C_{1}=\left(v_{1}, e_{1}, v_{2}, \cdots\right.$, $\left.e_{m}, v_{1}\right)$ and $C_{2}=\left(w_{1}, f_{1}, w_{2}, \cdots, f_{m}, w_{1}\right)$ are called equivalent if $w_{j}=v_{j+k}$ and $f_{j}=e_{j+k}$ for all $j$. Let $[C]$ be the equivalence class which contains a cycle $C$. Let $B^{r}$ be the cycle obtained by going $r$ times around a cycle $B$. Such a cycle is called a multiple of $B$. A cycle $C$ is reduced if both $C$ and $C^{2}$ have no hyperedge backtracking. Furthermore, a cycle $C$ is prime if it is not a multiple of a strictly smaller cycle.

The Ihara-Selberg zeta function of $H$ is defined by

$$
\zeta_{H}(t)=\prod_{[C]}\left(1-t^{|C|}\right)^{-1},
$$

where $[C]$ runs over all equivalence classes of prime, reduced cycles of $H$, and $t$ is a complex variable with $|t|$ sufficiently small(see [10]).

Let $H$ be a hypergraph with $E(H)=\left\{e_{1}, \ldots, e_{m}\right\}$, and let $\left\{c_{1}, \ldots, c_{m}\right\}$ be a set of $m$ colors, where $c\left(e_{i}\right)=c_{i}$. Then an edge-colored graph $G H_{c}$ is defined as a graph with vertex set $V(H)$ and edge set $\{v w \mid v, w \in V(H) ; v \neq w ; v, w \in e \in E(H)\}$, where an edge $v w$ is colored $c_{i}$ if $v, w \in e_{i}$. Note that $G H_{c}$ is identified with the "undirected" halved graph $B_{H}^{[1]}$ with colors. 
Let $G H_{c}^{o}$ be the symmetric digraph corresponding to the edge-clored graph $G H_{c}$. Then the oriented line graph $H_{L}^{o}=\left(V_{L}, E_{L}^{o}\right)$ associated with $G H_{c}^{o}$ by

$$
V_{L}=A\left(G H_{c}^{o}\right), \quad \text { and } \quad E_{L}^{o}=\left\{\left(e_{i}, e_{j}\right) \in A\left(G H_{c}^{o}\right) \times A\left(G H_{c}^{o}\right) \mid c\left(e_{i}\right) \neq c\left(e_{j}\right), t\left(e_{i}\right)=o\left(e_{j}\right)\right\} \text {, }
$$

where $c\left(e_{i}\right)$ is the same color as the one of the corresponding undirected edge in $D\left(G H_{c}^{o}\right)$. Also, $H_{L}^{o}$ is called the oriented line graph of $G H_{c}$. The Perron-Frobenius operator $\mathbf{T}$ : $C\left(V_{L}\right) \longrightarrow C\left(V_{L}\right)$ is given by

$$
(\mathbf{T} f)(x)=\sum_{e \in E_{o}(x)} f(t(e))
$$

where $E_{o}(x)=\left\{e \in E_{L}^{o} \mid o(e)=x\right\}$ is the set of all oriented edges with $x$ as their origin vertex, and $C\left(V_{L}\right)$ is the set of functions from $V_{L}$ to the complex number field $\mathbf{C}$.

Storm [10] gave two nice determinant expressions of the Ihara-Selberg zeta function of a hypergraph by using the results of Kotani and Sunada [7], and Bass [2].

Theorem 3 (Storm) Let $H$ be a finite, connected hypergraph such that every hypervetex is in at least two hyperedges. Then

$$
\begin{gathered}
\zeta_{H}(t)^{-1}=\operatorname{det}(\mathbf{I}-t \mathbf{T}) \\
=\mathbf{Z}\left(B_{H}, \sqrt{t}\right)^{-1}=(1-t)^{m-n} \operatorname{det}\left(\mathbf{I}-\sqrt{t} \mathbf{A}\left(B_{H}\right)+t \mathbf{Q}_{B_{H}}\right),
\end{gathered}
$$

where $n=\left|V\left(B_{H}\right)\right|, m=\left|E\left(B_{H}\right)\right|$ and $\mathbf{Q}_{B_{H}}=\mathbf{D}_{B_{H}}-\mathbf{I}$.

In Theorem 3, can the equality between the first identity (1) and the second identity (2) be proved by an analogue of Bass' method?

In Section 2, we present a new determinant expression for the Ihara-Selberg zeta function of a hypergraph. In Section 3, we show that, in Theorem 3, the first identity (1) is obtained from the second identity (2) by using a linear algebraic method. In Section 4, we generalize theses results to the Bartholdi zeta function of a hypergraph.

\section{A new determinant expression of the zeta function of a hypergraph}

Let $H=(V(H), E(H))$ be a hypergraph, $V(H)=\left\{v_{1}, \ldots, v_{n}\right\}$ and $E(H)=\left\{e_{1}, \ldots, e_{m}\right\}$. Let $B_{H}$ have $\nu$ vertices and $\epsilon$ edges, where $\nu=n+m$. Then we have

$$
D\left(B_{H}\right)=\{(v, e),(e, v) \mid v \in e, v \in V(H), e \in E(H)\} .
$$

Let $f_{1}, \ldots, f_{\epsilon}$ be arcs in $B_{H}$ such that $o\left(f_{i}\right) \in V(H)$ for each $i=1, \ldots, \epsilon$. Then two $\epsilon \times \epsilon$ matrices $\mathbf{X}=\left(X_{i j}\right)$ and $\mathbf{Y}=\left(Y_{i j}\right)$ are defined as follows:

$$
X_{i j}= \begin{cases}1 & \text { if there exists an arc } f_{k}^{-1} \text { such that }\left(f_{i}, f_{k}^{-1}, f_{j}\right) \text { is a reduced path } \\ 0 & \text { otherwise }\end{cases}
$$


and

$Y_{i j}= \begin{cases}1 & \text { if there exists an arc } f_{k} \text { such that }\left(f_{i}^{-1}, f_{k}, f_{j}^{-1}\right) \text { is a reduced path } \\ 0 & \text { otherwise. }\end{cases}$

Remark that $\mathbf{Y}={ }^{t} \mathbf{X}$.

Theorem 4 Let $H$ be a finite, connected hypergraph such that every hypervetex is in at least two hyperedges. Set $\epsilon=\left|E\left(B_{H}\right)\right|$. Then

$$
\mathbf{Z}\left(B_{H}, \sqrt{t}\right)^{-1}=\operatorname{det}\left(\mathbf{I}_{\epsilon}-t \mathbf{X}\right)=\operatorname{det}\left(\mathbf{I}_{\epsilon}-t \mathbf{Y}\right)
$$

Proof. Let $H=(V(H), E(H))$ be a hypergraph, $V(H)=\left\{v_{1}, \ldots, v_{n}\right\}$ and $E(H)=$ $\left\{e_{1}, \ldots, e_{m}\right\}$. Let $B_{H}$ have $\nu$ vertices and $\epsilon$ edges. By Theorem 1 , we have

$$
\begin{aligned}
\mathbf{Z}\left(B_{H}, \sqrt{t}\right)^{-1} & =(1-t)^{\epsilon-\nu} \operatorname{det}\left(\mathbf{I}_{\nu}-\sqrt{t} \mathbf{A}\left(B_{H}\right)+t\left(\mathbf{D}_{B_{H}}-\mathbf{I}_{\nu}\right)\right) \\
& =\operatorname{det}\left(\mathbf{I}_{2 \epsilon}-\sqrt{t}\left(\mathbf{B}\left(B_{H}\right)-\mathbf{J}_{0}\left(B_{H}\right)\right)\right)
\end{aligned}
$$

Arrange arcs of $B_{H}$ as follows: $f_{1}, \ldots, f_{\epsilon}, f_{1}^{-1}, \ldots, f_{\epsilon}^{-1}$. We consider two matrices $\mathbf{B}$ and $\mathbf{J}_{0}$ under this order. Let

$$
\mathbf{B}\left(B_{H}\right)-\mathbf{J}_{0}\left(B_{H}\right)=\left[\begin{array}{cc}
\mathbf{0} & \mathbf{F} \\
\mathbf{G} & \mathbf{0}
\end{array}\right] .
$$

It is clear that both $\mathbf{F}$ and $\mathbf{G}$ are symmetric, but $\mathbf{F} \neq{ }^{t} \mathbf{G}$. Furthermore,

$$
\mathbf{F G}=\mathbf{X} \text { and } \mathbf{G F}=\mathbf{Y}
$$

Thus, we have

$$
\begin{aligned}
\operatorname{det}\left(\mathbf{I}_{2 \epsilon}-\sqrt{t}\left(\mathbf{B}\left(B_{H}\right)-\mathbf{J}_{0}\left(B_{H}\right)\right)\right) & =\operatorname{det}\left(\left[\begin{array}{cc}
\mathbf{I}_{\epsilon} & -\sqrt{t} \mathbf{F} \\
-\sqrt{t} \mathbf{G} & \mathbf{I}_{\epsilon}
\end{array}\right]\right) \\
& =\operatorname{det}\left(\left[\begin{array}{cc}
\mathbf{I}_{\epsilon}-t \mathbf{F G} & -\sqrt{t} \mathbf{F} \\
\mathbf{0} & \mathbf{I}_{\epsilon}
\end{array}\right]\right) \\
& =\operatorname{det}\left(\mathbf{I}_{\epsilon}-t \mathbf{F G}\right)=\operatorname{det}\left(\mathbf{I}_{\epsilon}-t \mathbf{X}\right) \\
& =\operatorname{det}\left(\mathbf{I}_{\epsilon}-t \mathbf{G F}\right)=\operatorname{det}\left(\mathbf{I}_{\epsilon}-t \mathbf{Y}\right)
\end{aligned}
$$

Therefore, the result follows. Q.E.D. 


\section{A linear algebraic proof of Storm Theorem}

We show that, in Theorem 3, the identity (1) is obtained from the identity (2) by using a linear algebraic method.

Let $H=(V(H), E(H))$ be a hypergraph, $V(H)=\left\{v_{1}, \ldots, v_{n}\right\}$ and $E(H)=\left\{e_{1}, \ldots\right.$, $\left.e_{m}\right\}$. Let $B_{H}$ have $\nu$ vertices and $\epsilon$ edges, and $D\left(B_{H}\right)=\left\{f_{1}, \ldots, f_{\epsilon}, f_{1}^{-1}, \ldots, f_{\epsilon}^{-1}\right\}$ such that $o\left(f_{i}\right) \in V(H)(1 \leqslant i \leqslant \epsilon)$. Furthermore, let $\mathcal{R}$ (or $\mathcal{S}$ ) be the set of reduced paths $P$ in $B_{H}$ with length two such that $o(P), t(P) \in V(H)($ or $o(P), t(P) \in E(H))$. Set $r=|\mathcal{R}|$ and $s=|\mathcal{S}|$. For a path $P=(x, y, z)$ of length two in $B_{H}$, let

$$
\text { oe }(P)=(x, y), t e(P)=(y, z),
$$

where $(x, y, z)=(v, e, w)$ or $(x, y, z)=(e, v, f)(v, w \in V(H) ; e, f \in E(H))$.

Now, we introduce two $r \times \epsilon$ matrices $\mathbf{K}=\left(K_{P f_{j}^{-1}}\right)_{P \in R ; 1 \leqslant j \leqslant \epsilon}$ and $\mathbf{L}=\left(L_{P f_{j}}\right)_{P \in R ; 1 \leqslant j \leqslant \epsilon}$ are defined as follows:

$$
K_{P f_{j}^{-1}}=\left\{\begin{array}{ll}
1 & \text { if } t e(P)=f_{j}^{-1} \\
0 & \text { otherwise }
\end{array}, \quad L_{P f_{j}}= \begin{cases}1 & \text { if } o e(P)=f_{j} \\
0 & \text { otherwise }\end{cases}\right.
$$

Furthermore, two $s \times \epsilon$ matrices $\mathbf{M}=\left(M_{Q f_{j}^{-1}}\right)_{Q \in S ; 1 \leqslant j \leqslant \epsilon}$ and $\mathbf{N}=\left(N_{Q f_{j}}\right)_{Q \in S ; 1 \leqslant j \leqslant \epsilon}$ are defined as follows:

$$
M_{Q f_{j}^{-1}}=\left\{\begin{array}{l}
1 \text { if } o e(Q)=f_{j}^{-1}, \\
0 \text { otherwise, }
\end{array} \quad N_{Q f_{j}}= \begin{cases}1 & \text { if } t e(Q)=f_{j}, \\
0 & \text { otherwise. }\end{cases}\right.
$$

Then we have

$$
{ }^{t} \mathbf{L K}=\mathbf{F} \text { and }{ }^{t} \mathbf{M N}=\mathbf{G} .
$$

and, $\mathbf{K}^{t} \mathbf{M}=\left(b_{P Q}\right)_{P \in \mathcal{R} ; Q \in \mathcal{S}}$ and $\mathbf{N}^{t} \mathbf{L}=\left(c_{Q P}\right)_{P \in \mathcal{R} ; Q \in \mathcal{S}}$ are given as follows:

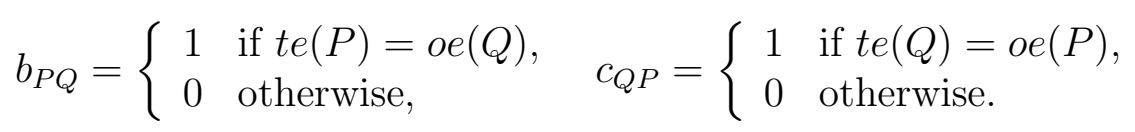

Thus, we have

$$
\mathbf{K}^{t} \mathbf{M N}{ }^{t} \mathbf{L}=\mathbf{T}
$$

Furthermore, by (3) and (4),

$$
{ }^{t} \mathbf{L K}{ }^{t} \mathbf{M N}=\mathbf{F G}=\mathbf{X} .
$$

Here it is known that, for a $m \times n$ matrix $\mathbf{A}$ and $n \times m$ matrix $\mathbf{B}$,

$$
\operatorname{det}\left(\mathbf{I}_{m}+\mathbf{A B}\right)=\operatorname{det}\left(\mathbf{I}_{n}+\mathbf{B A}\right) .
$$

Therefore, it follows that

$$
\operatorname{det}\left(\mathbf{I}_{r}-t \mathbf{T}\right)=\operatorname{det}\left(\mathbf{I}_{\epsilon}-t \mathbf{X}\right) .
$$

By Theorem 4 and the fact that $\zeta_{H}(t)^{-1}=\mathbf{Z}\left(B_{H}, \sqrt{t}\right)^{-1}$, we have

$$
\zeta_{H}(t)^{-1}=\operatorname{det}\left(\mathbf{I}_{r}-t \mathbf{T}\right)
$$

Q.E.D. 


\section{Bartholdi zeta function of a hypergraph}

Let $H$ be a hypergraph. Then a path $P=\left(v_{1}, e_{1}, v_{2}, e_{2}, \cdots, e_{n}, v_{n+1}\right)$ has a (broad) backtracking or (broad) bump at $e$ or $v$ if there is a subsequence of $P$ of the form $(e, v, e)$ or $(v, e, v)$, where $e \in E(H), v \in V(H)$. Furthermore, the cyclic bump count $\operatorname{cbc}(C)$ of a cycle $C=\left(v_{1}, e_{1}, v_{2}, e_{2}, \cdots, e_{n}, v_{1}\right)$ is

$$
\operatorname{cbc}(C)=\left|\left\{i=1, \cdots, n \mid v_{i}=v_{i+1}\right\}\right|+\left|\left\{i=1, \cdots, n \mid e_{i}=e_{i+1}\right\}\right|,
$$

where $v_{n+1}=v_{1}$ and $e_{n+1}=e_{1}$.

The Bartholdi zeta function of $H$ is defined by

$$
\zeta(H, u, t)=\prod_{[C]}\left(1-u^{c b c(C)} t^{|C|}\right)^{-1}
$$

where $[C]$ runs over all equivalence classes of prime cycles of $H$, and $u, t$ are complex variables with $|u|,|t|$ sufficiently small.

If $u=0$, then the Bartholdi zeta function of $H$ is the Ihara-Selberg zeta function of $H$.

Sato [8] presented a determinant expression of the Bartholdi zeta function of a hypergraph.

Theorem 5 (Sato) Let $H$ be a finite, connected hypergraph such that every hypervetex is in at least two hyperedges. Then

$$
\begin{aligned}
\zeta(H, u, t)^{-1} & =\zeta\left(B_{H}, u, \sqrt{t}\right)^{-1} \\
& =\left(1-(1-u)^{2} t\right)^{m-n} \operatorname{det}\left(\mathbf{I}-\sqrt{t} \mathbf{A}\left(B_{H}\right)+(1-u) t\left(\mathbf{D}_{B_{H}}-(1-u) \mathbf{I}\right)\right),
\end{aligned}
$$

where $n=\left|V\left(B_{H}\right)\right|$ and $m=\left|E\left(B_{H}\right)\right|$.

Let $H=(V(H), E(H))$ be a hypergraph, $V(H)=\left\{v_{1}, \ldots, v_{n}\right\}$ and $E(H)=\left\{e_{1}, \ldots\right.$, $\left.e_{m}\right\}$. Let $B_{H}$ have $\nu$ vertices and $\epsilon$ edges, $V_{1}=V(H)$ and $V_{2}=E(H)$. Then, the broad halved graph $B_{H}^{(i)}$ of $B_{H}$ is defined to be the graph with vertex set $V_{i}$ and arc set $\left\{P\right.$ : path ||$\left.P \mid=2 ; o(P), t(P) \in V_{i}\right\}$ for $i=1,2$. Furthermore, let $\left\{c_{1}, \ldots, c_{m}\right\}$ be a set of $m$ colors such that $c\left(e_{i}\right)=c_{i}$ for $i=1, \ldots, m$. We color each arc of $B_{H}^{(1)}$ as follows:

$$
c(P)=c(e) \text { for } P=(v, e, w) \in D\left(B_{H}^{(1)}\right) .
$$

Then the line digraph $\vec{L}\left(B_{H}^{(1)}\right)$ of $B_{H}^{(1)}$ is defined as follows: $V\left(\vec{L}\left(B_{H}^{(1)}\right)\right)=D\left(B_{H}^{(1)}\right)$, and $(P, Q) \in A\left(\vec{L}\left(B_{H}^{(1)}\right)\right)$ if and only if $t(P)=o(Q)$ in $B_{H}$.

Next, let $\mathcal{R}^{\prime}$ (or $\mathcal{S}^{\prime}$ ) be the set of paths $P$ in $B_{H}$ with length two such that $o(P), t(P) \in$ $V(H)$ ( or $\in E(H)$ ). Furthermore, let $f_{k}=\left(v_{i_{k}}, e_{j_{k}}\right), P_{k}=\left(v_{i_{k}}, e_{j_{k}}, v_{i_{k}}\right)$ and $Q_{k}=$ $\left(e_{j_{k}}, v_{i_{k}}, e_{j_{k}}\right)$ for each $k=1, \ldots, \epsilon$. Then we have

$$
\mathcal{R}^{\prime}=\mathcal{R} \cup\left\{P_{1}, \ldots, P_{\epsilon}\right\} \text { and } \mathcal{S}^{\prime}=\mathcal{S} \cup\left\{Q_{1}, \ldots, Q_{\epsilon}\right\}
$$


Furthermore, we have $\left|\mathcal{R}^{\prime}\right|=r+\epsilon$ and $\left|\mathcal{S}^{\prime}\right|=s+\epsilon$.

Now, we introduce a $(r+\epsilon) \times(r+\epsilon)$ matrix $\mathbf{T}^{\prime}=\left(T_{P P^{\prime}}^{\prime}\right)_{P, P^{\prime} \in \mathcal{R}^{\prime}}$ for the line digraph $\vec{L}\left(B_{H}^{(1)}\right)$ of the halved graph $B_{H}^{(1)}$ is defined as follows:

$$
T_{P P^{\prime}}^{\prime}= \begin{cases}u^{2} & \text { if } t(P)=o\left(P^{\prime}\right), P=P^{\prime} \in \mathcal{R}^{\prime} \backslash \mathcal{R}, \\ u^{2} & \text { if } t(P)=o\left(P^{\prime}\right), P \in \mathcal{R}^{\prime} \backslash \mathcal{R}, P^{\prime} \in \mathcal{R} \text { and } c(P)=c\left(P^{\prime}\right), \\ u & \text { if } t(P)=o\left(P^{\prime}\right), P, P^{\prime} \in \mathcal{R}^{\prime} \backslash \mathcal{R} \text { and } c(P) \neq c\left(P^{\prime}\right), \\ u & \text { if } t(P)=o\left(P^{\prime}\right), P \in \mathcal{R}^{\prime} \backslash \mathcal{R}, P^{\prime} \in \mathcal{R} \text { and } c(P) \neq c\left(P^{\prime}\right), \\ u & \text { if } t(P)=o\left(P^{\prime}\right), P \in \mathcal{R}, P^{\prime} \in \mathcal{R}^{\prime} \backslash \mathcal{R} \text { and } c(P)=c\left(P^{\prime}\right), \\ u & \text { if } t(P)=o\left(P^{\prime}\right), P, P^{\prime} \in \mathcal{R} \text { and } c(P)=c\left(P^{\prime}\right), \\ 1 & \text { if } t(P)=o\left(P^{\prime}\right), P \in \mathcal{R}, P^{\prime} \in \mathcal{R}^{\prime} \backslash \mathcal{R} \text { and } c(P) \neq c\left(P^{\prime}\right), \\ 1 & \text { if } t(P)=o\left(P^{\prime}\right), P, P^{\prime} \in \mathcal{R} \text { and } c(P) \neq c\left(P^{\prime}\right), \\ 0 & \text { otherwise, }\end{cases}
$$

We present a new determinant expression for the Bartholdi zeta function of a hypergraph.

Theorem 6 Let $H$ be a finite, connected hypergraph such that every hypervetex is in at least two hyperedges. Set $\epsilon=\left|E\left(B_{H}\right)\right|$ and $r=|\mathcal{R}|$. Then

$$
\begin{gathered}
\zeta(H, u, t)^{-1}=\operatorname{det}\left(\mathbf{I}_{r+\epsilon}-t \mathbf{T}^{\prime}\right) \\
=\operatorname{det}\left(\mathbf{I}_{\epsilon}-t\left(\mathbf{X}+u(\mathbf{F}+\mathbf{G})+u^{2} \mathbf{I}_{\epsilon}\right)\right)=\operatorname{det}\left(\mathbf{I}_{\epsilon}-t\left(\mathbf{Y}+u(\mathbf{F}+\mathbf{G})+u^{2} \mathbf{I}_{\epsilon}\right)\right) .
\end{gathered}
$$

Proof. Let $H=(V(H), E(H))$ be a hypergraph, $V(H)=\left\{v_{1}, \ldots, v_{n}\right\}$ and $E(H)=$ $\left\{e_{1}, \ldots, e_{m}\right\}$. Let $B_{H}$ have $\nu$ vertices and $\epsilon$ edges. By Theorems 2 and 5 , we have

$$
\begin{gathered}
\zeta(H, u, t)^{-1}=\operatorname{det}\left(\mathbf{I}_{2 \epsilon}-\sqrt{t}\left(\mathbf{B}\left(B_{H}\right)-(1-u) \mathbf{J}_{0}\left(B_{H}\right)\right)\right) \\
=\left(1-(1-u)^{2} t\right)^{\epsilon-\nu} \operatorname{det}\left(\mathbf{I}_{\nu}-\sqrt{t} \mathbf{A}\left(B_{H}\right)+(1-u) t\left(\mathbf{D}_{B_{H}}-(1-u) \mathbf{I}_{\nu}\right)\right) .
\end{gathered}
$$

Arrange $\operatorname{arcs}$ of $B_{H}$ as follows: $f_{1}, \ldots, f_{\epsilon}, f_{1}^{-1}, \ldots, f_{\epsilon}^{-1}$ such that $o\left(f_{i}\right) \in V(H)(1 \leqslant$ $i \leqslant \epsilon)$. We consider two matrices $\mathbf{B}$ and $\mathbf{J}_{0}$ under this order. Let

$$
\mathbf{B}\left(B_{H}\right)-(1-u) \mathbf{J}_{0}\left(B_{H}\right)=\left[\begin{array}{cc}
\mathbf{0} & \mathbf{F}+u \mathbf{I}_{\epsilon} \\
\mathbf{G}+u \mathbf{I}_{\epsilon} & \mathbf{0}
\end{array}\right] .
$$

Thus, by (3), we have

$$
\begin{aligned}
& \operatorname{det}\left(\mathbf{I}_{2 \epsilon}-\sqrt{t}\left(\mathbf{B}\left(B_{H}\right)-(1-u) \mathbf{J}_{0}\left(B_{H}\right)\right)\right) \\
& =\operatorname{det}\left(\left[\begin{array}{cc}
\mathbf{I}_{\epsilon} & -\sqrt{t}\left(\mathbf{F}+u \mathbf{I}_{\epsilon}\right) \\
-\sqrt{t}\left(\mathbf{G}+u \mathbf{I}_{\epsilon}\right) & \mathbf{I}_{\epsilon}
\end{array}\right]\right) \\
& =\operatorname{det}\left(\left[\begin{array}{cc}
\mathbf{I}_{\epsilon}-t\left(\mathbf{F}+u \mathbf{I}_{\epsilon}\right)\left(\mathbf{G}+u \mathbf{I}_{\epsilon}\right) & -\sqrt{t}\left(\mathbf{F}+u \mathbf{I}_{\epsilon}\right) \\
\mathbf{0} & \mathbf{I}_{\epsilon}
\end{array}\right]\right) \\
& =\operatorname{det}\left(\mathbf{I}_{\epsilon}-t\left(\mathbf{F G}+u(\mathbf{F}+\mathbf{G})+u^{2} \mathbf{I}_{\epsilon}\right)\right)=\operatorname{det}\left(\mathbf{I}_{\epsilon}-t\left(\mathbf{X}+u(\mathbf{F}+\mathbf{G})+u^{2} \mathbf{I}_{\epsilon}\right)\right) \\
& =\operatorname{det}\left(\mathbf{I}_{\epsilon}-t\left(\mathbf{G F}+u(\mathbf{F}+\mathbf{G})+u^{2} \mathbf{I}_{\epsilon}\right)\right)=\operatorname{det}\left(\mathbf{I}_{\epsilon}-t\left(\mathbf{Y}+u(\mathbf{F}+\mathbf{G})+u^{2} \mathbf{I}_{\epsilon}\right)\right) .
\end{aligned}
$$


Arrange elements of $\mathcal{R}^{\prime}$ and $\mathcal{S}^{\prime}$ as follows:

$$
P_{1}, \ldots, P_{\epsilon}, \mathcal{R} ; Q_{1}, \ldots, Q_{\epsilon}, \mathcal{S}
$$

where $P_{k}=\left(v_{i_{k}}, e_{j_{k}}, v_{i_{k}}\right)$ and $Q_{k}=\left(e_{j_{k}}, v_{i_{k}}, e_{j_{k}}\right)$ if $f_{k}=\left(v_{i_{k}}, e_{j_{k}}\right)$ for $k=1, \ldots, \epsilon$. Then we introduce two $(r+\epsilon) \times \epsilon$ matrices $\mathbf{K}^{\prime}=\left(K_{P f_{j}^{-1}}^{\prime}\right)_{P \in \mathcal{R}^{\prime} ; 1 \leqslant j \leqslant \epsilon}$ and $\mathbf{L}^{\prime}=\left(L_{P f_{j}}^{\prime}\right)_{P \in \mathcal{R}^{\prime} ; 1 \leqslant j \leqslant \epsilon}$ are defined as follows:

$$
K_{P f_{j}^{-1}}^{\prime}=\left\{\begin{array}{l}
1 \text { if } t e(P)=f_{j}^{-1} \text { and } t e(P) \neq o e(P)^{-1}, \\
u \text { if } t e(P)=o e(P)^{-1}=f_{j}^{-1}, \\
0 \quad \text { otherwise, }
\end{array} \quad L_{P f_{j}}^{\prime}= \begin{cases}1 & \text { if } o e(P)=f_{j}, \\
0 & \text { otherwise }\end{cases}\right.
$$

Furthermore, two $(s+\epsilon) \times \epsilon$ matrices $\mathbf{M}^{\prime}=\left(M_{Q f_{j}^{-1}}^{\prime}\right)_{Q \in \mathcal{S}^{\prime} ; 1 \leqslant j \leqslant \epsilon}$ and $\mathbf{N}^{\prime}=\left(N_{Q f_{j}}^{\prime}\right)_{Q \in \mathcal{S}^{\prime} ; 1 \leqslant j \leqslant \epsilon}$ are defined as follows:

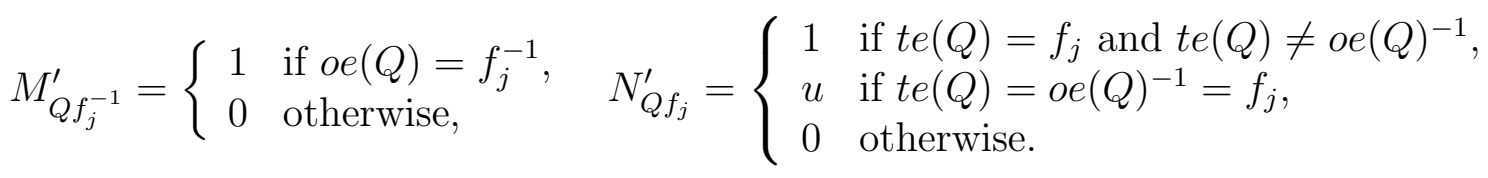

Here we have

$$
\mathbf{K}^{\prime}=\left[\begin{array}{c}
u \mathbf{I}_{\epsilon} \\
\mathbf{K}
\end{array}\right], \mathbf{L}^{\prime}=\left[\begin{array}{c}
\mathbf{I}_{\epsilon} \\
\mathbf{L}
\end{array}\right], \mathbf{M}^{\prime}=\left[\begin{array}{c}
\mathbf{I}_{\epsilon} \\
\mathbf{M}
\end{array}\right] \text { and } \mathbf{N}^{\prime}=\left[\begin{array}{c}
u \mathbf{I}_{\epsilon} \\
\mathbf{N}
\end{array}\right] .
$$

Thus, we have

$$
\mathbf{K}^{\prime t} \mathbf{M}^{\prime} \mathbf{N}^{\prime t} \mathbf{L}^{\prime}=\left[\begin{array}{cc}
u^{2} \mathbf{I}_{\epsilon}+u{ }^{t} \mathbf{M N} & u^{2}{ }^{t} \mathbf{L}+u{ }^{t} \mathbf{M} \mathbf{N}{ }^{t} \mathbf{L} \\
u \mathbf{K}+\mathbf{K}{ }^{t} \mathbf{M N} & u \mathbf{K}{ }^{t} \mathbf{L}+\mathbf{K}{ }^{t} \mathbf{M} \mathbf{N}{ }^{t} \mathbf{L}
\end{array}\right]
$$

A nonzero element of $u^{2} \mathbf{I}_{\epsilon}, u^{t} \mathbf{M N}, u^{2}{ }^{t} \mathbf{L}, u^{t} \mathbf{M N}{ }^{t} \mathbf{L}, u \mathbf{K}, \mathbf{K}{ }^{t} \mathbf{M N}, u \mathbf{K}{ }^{t} \mathbf{L}$ and $\mathbf{K}{ }^{t} \mathbf{M} \mathbf{N}^{t} \mathbf{L}$ corresponds to a sequence of eight paths of length two, respectively:

$$
\begin{gathered}
P_{i} \rightarrow Q_{i} \rightarrow P_{i} ; P_{i} \rightarrow Q \rightarrow P_{j}\left(c\left(P_{i}\right) \neq c\left(P_{j}\right)\right) ; P_{i} \rightarrow Q_{i} \rightarrow R\left(c\left(P_{i}\right)=c(R)\right) ; \\
P_{i} \rightarrow Q \rightarrow R\left(c\left(P_{i}\right) \neq c(R)\right) ; P \rightarrow Q_{i} \rightarrow P_{i}\left(c(P)=c\left(P_{i}\right)\right) ; P \rightarrow Q \rightarrow P_{i}\left(c(P) \neq c\left(P_{i}\right)\right) ; \\
P \rightarrow Q_{i} \rightarrow R(c(P)=c(R)) ; P \rightarrow Q \rightarrow R(c(P) \neq c(R)),
\end{gathered}
$$

where $P, R \in \mathcal{R}, Q \in \mathcal{S}, i=1, \ldots, \epsilon$, and the notation $P \rightarrow Q$ implies that $\operatorname{te}(P)=o e(Q)$ in $B_{H}$. Therefore, it follows that

$$
\mathbf{K}^{\prime t} \mathbf{M}^{\prime} \mathbf{N}^{\prime t} \mathbf{L}^{\prime}=\mathbf{T}^{\prime}
$$

By (3) and (4), we have

$$
{ }^{t} \mathbf{L}^{\prime} \mathbf{K}^{\prime}{ }^{t} \mathbf{M}^{\prime} \mathbf{N}^{\prime}=u^{2} \mathbf{I}_{\epsilon}+u{ }^{t} \mathbf{L K}+u{ }^{t} \mathbf{M N}+{ }^{t} \mathbf{L} \mathbf{K}{ }^{t} \mathbf{M N}=u^{2} \mathbf{I}_{\epsilon}+u(\mathbf{F}+\mathbf{G})+\mathbf{X} .
$$

By (6),(8) and (9), it follows that

$$
\operatorname{det}\left(\mathbf{I}_{r+\epsilon}-t \mathbf{T}^{\prime}\right)=\operatorname{det}\left(\mathbf{I}_{\epsilon}-t\left(\mathbf{X}+u(\mathbf{F}+\mathbf{G})+u^{2} \mathbf{I}_{\epsilon}\right)\right) .
$$

Q.E.D.

If $u=0$, then Theorem 6 implies (1) of Theorem 3 . 
Corollary 1 Let $H$ be a finite, connected hypergraph such that every hypervetex is in at least two hyperedges. Set $r=|\mathcal{R}|$. Then

$$
\zeta_{H}(t)^{-1}=\operatorname{det}\left(\mathbf{I}_{r}-t \mathbf{T}\right) .
$$

Proof. Set $\epsilon=\left|E\left(B_{H}\right)\right|$ and $u=0$. By Theorem 6 and (5), (7), we have

$$
\zeta_{H}(t)^{-1}=\operatorname{det}\left(\mathbf{I}_{r+\epsilon}-t \mathbf{T}^{\prime}\right)=\operatorname{det}\left(\left[\begin{array}{cc}
\mathbf{I}_{\epsilon} & \mathbf{0} \\
-t \mathbf{K}^{t} \mathbf{M N} & \mathbf{I}_{r}-t \mathbf{T}
\end{array}\right]\right)=\operatorname{det}\left(\mathbf{I}_{r}-t \mathbf{T}\right) .
$$

Q.E.D.

\section{Example}

Let $H$ be the hypergraph with $V(H)=\left\{v_{1}, v_{2}, v_{3}\right\}$ and $E(H)=\left\{e_{1}, e_{2}, e_{3}\right\}$, where $e_{1}=$ $\left\{v_{1}, v_{2}\right\}, e_{2}=\left\{v_{1}, v_{3}\right\}$ and $e_{3}=\left\{v_{1}, v_{2}, v_{3}\right\}$. Furthermore, let $B_{H}$ be the bipartite graph associated with $H$. Let $f_{1}=\left(v_{1}, e_{1}\right), f_{2}=\left(v_{1}, e_{2}\right), f_{3}=\left(v_{1}, e_{3}\right), f_{4}=\left(v_{2}, e_{1}\right), f_{5}=\left(v_{2}, e_{3}\right)$, $f_{6}=\left(v_{3}, e_{2}\right)$ and $f_{7}=\left(v_{3}, e_{3}\right)$. Then we have $D\left(B_{H}\right)=\left\{f_{1}, \ldots, f_{7}, f_{1}^{-1}, \ldots, f_{7}^{-1}\right\}$. The matrices $\mathbf{X}$ is given as follows:

$$
\mathbf{X}=\left[\begin{array}{lllllll}
0 & 0 & 0 & 0 & 1 & 0 & 0 \\
0 & 0 & 0 & 0 & 0 & 0 & 1 \\
0 & 0 & 0 & 1 & 0 & 1 & 0 \\
0 & 1 & 1 & 0 & 0 & 0 & 0 \\
1 & 1 & 0 & 0 & 0 & 1 & 0 \\
1 & 0 & 1 & 0 & 0 & 0 & 0 \\
1 & 1 & 0 & 1 & 0 & 0 & 0
\end{array}\right]
$$

By Theorem 4, we have

$$
\zeta(H, t)^{-1}=\operatorname{det}\left(\mathbf{I}_{7}-t \mathbf{X}\right)=(1-t)\left(1+t+t^{2}\right)\left(1-4 t^{2}-t^{3}+4 t^{4}\right) .
$$

Next, two matrices $\mathbf{F}$ and $\mathbf{G}$ are given as follows:

$$
\mathbf{F}=\left[\begin{array}{lllllll}
0 & 0 & 0 & 1 & 0 & 0 & 0 \\
0 & 0 & 0 & 0 & 0 & 1 & 0 \\
0 & 0 & 0 & 0 & 1 & 0 & 1 \\
1 & 0 & 0 & 0 & 0 & 0 & 0 \\
0 & 0 & 1 & 0 & 0 & 0 & 1 \\
0 & 1 & 0 & 0 & 0 & 0 & 0 \\
0 & 0 & 1 & 0 & 1 & 0 & 0
\end{array}\right], \mathbf{G}=\left[\begin{array}{lllllll}
0 & 1 & 1 & 0 & 0 & 0 & 0 \\
1 & 0 & 1 & 0 & 0 & 0 & 0 \\
1 & 1 & 0 & 0 & 0 & 0 & 0 \\
0 & 0 & 0 & 0 & 1 & 0 & 0 \\
0 & 0 & 0 & 1 & 0 & 0 & 0 \\
0 & 0 & 0 & 0 & 0 & 0 & 1 \\
0 & 0 & 0 & 0 & 0 & 1 & 0
\end{array}\right]
$$

Then it is certain that $\mathbf{F G}=\mathbf{X}$. 
Furthermore,

$$
\mathbf{X}+u \mathbf{F}+u \mathbf{G}+u^{2} \mathbf{I}_{7}=\left[\begin{array}{ccccccc}
u^{2} & u & u & u & 1 & 0 & 0 \\
u & u^{2} & u & 0 & 0 & u & 1 \\
u & u & u^{2} & 1 & u & 1 & u \\
u & 1 & 1 & u^{2} & u & 0 & 0 \\
1 & 1 & u & u & u^{2} & 1 & u \\
1 & u & 1 & 0 & 0 & u^{2} & u \\
1 & 1 & u & 1 & u & u & u^{2}
\end{array}\right]
$$

By Theorem 6, we have

$$
\begin{aligned}
& \zeta(H, u, t)^{-1}=\operatorname{det}\left(\mathbf{I}_{7}-t\left(\mathbf{X}+u \mathbf{F}+u \mathbf{G}+u^{2} \mathbf{I}_{7}\right)\right) \\
= & \left(1-(1-u)^{2} t\right)\left(1+\left(1-2 u^{2}\right) t+\left(1-u^{2}\right)^{2} t^{2}\right) \\
\times & \left(1-2 u(1+2 u) t+\left(-4-2 u-5 u^{2}-6 u^{3}+6 u^{4}\right) t^{2}\right. \\
- & \left.(1-u)^{2}\left(1+4 u+14 u^{2}+14 u^{3}+4 u^{4}\right) t^{3}+(1-u)^{4}(1+u)^{2}(2+u)^{2} t^{4}\right) .
\end{aligned}
$$

Now, we consider arcs of $B_{H}^{(1)}$. Let $R_{1}=\left(v_{1}, e_{1}, v_{2}\right), R_{2}=\left(v_{1}, e_{2}, v_{3}\right), R_{3}=\left(v_{1}, e_{3}, v_{2}\right)$, $R_{4}=\left(v_{1}, e_{3}, v_{3}\right), R_{5}=R_{1}^{-1}, R_{6}=R_{3}^{-1}, R_{7}=\left(v_{2}, e_{3}, v_{3}\right), R_{8}=R_{2}^{-1}, R_{9}=R_{4}^{-1}, R_{10}=$ $R_{7}^{-1}$ and $P_{i}=\left(f_{i}, f_{i}^{-1}\right)(1 \leqslant i \leqslant 7)$. Arrange elements of $\mathcal{R}^{\prime}=D\left(B_{H}^{(1)}\right)$ as follows: $P_{1}, \cdots, P_{7}, R_{1}, \cdots, R_{10}$. We consider the matrix $\mathbf{T}^{\prime}$ under this order, and then, we have

$$
\mathbf{T}^{\prime}=\left[\begin{array}{ccccccccccccccccc}
u^{2} & u & u & 0 & 0 & 0 & 0 & u^{2} & u & u & u & 0 & 0 & 0 & 0 & 0 & 0 \\
u & u^{2} & u & 0 & 0 & 0 & 0 & u & u^{2} & u & u & 0 & 0 & 0 & 0 & 0 & 0 \\
u & u & u^{2} & 0 & 0 & 0 & 0 & u & u & u^{2} & u^{2} & 0 & 0 & 0 & 0 & 0 & 0 \\
0 & 0 & 0 & u^{2} & u & 0 & 0 & 0 & 0 & 0 & 0 & u^{2} & u & u & 0 & 0 & 0 \\
0 & 0 & 0 & u & u^{2} & 0 & 0 & 0 & 0 & 0 & 0 & u & u^{2} & u^{2} & 0 & 0 & 0 \\
0 & 0 & 0 & 0 & 0 & u^{2} & u & 0 & 0 & 0 & 0 & 0 & 0 & 0 & u^{2} & u & u \\
0 & 0 & 0 & 0 & 0 & s & u^{2} & 0 & 0 & 0 & 0 & 0 & 0 & 0 & u & u^{2} & u^{2} \\
u & 1 & 1 & 0 & 0 & 0 & 0 & 0 & 0 & 0 & 0 & u & 1 & 1 & 0 & 0 & 0 \\
1 & u & 1 & 0 & 0 & 0 & 0 & 0 & 0 & 0 & 0 & 0 & 0 & 0 & u & 1 & 1 \\
1 & 1 & u & 0 & 0 & 0 & 0 & 0 & 0 & 0 & 0 & 1 & u & 0 & 0 & 0 & 0 \\
1 & 1 & u & 0 & 0 & 0 & 0 & 0 & 0 & 0 & 0 & 0 & 0 & 0 & 1 & u & 0 \\
0 & 0 & 0 & u & 1 & 0 & 0 & u & 1 & 1 & 1 & 0 & 0 & 0 & 0 & 0 & 0 \\
0 & 0 & 0 & 1 & u & 0 & 0 & 1 & 1 & u & 0 & 0 & 0 & 0 & 0 & 0 & 0 \\
0 & 0 & 0 & 1 & u & 0 & 0 & 0 & 0 & 0 & 0 & 0 & 0 & 0 & 1 & 0 & u \\
0 & 0 & 0 & 0 & 0 & u & 1 & 1 & u & 1 & 1 & 0 & 0 & 0 & 0 & 0 & 0 \\
0 & 0 & 0 & 0 & 0 & 1 & u & 1 & 1 & 0 & u & 0 & 0 & 0 & 0 & 0 & 0 \\
0 & 0 & 0 & 0 & 0 & 1 & u & 0 & 0 & 0 & 0 & 1 & 0 & u & 0 & 0 & 0
\end{array}\right] .
$$

By Theorem 6, we have

$$
\operatorname{det}\left(\mathbf{I}_{17}-t \mathbf{T}^{\prime}\right)=\zeta(H, u, t)^{-1}
$$


Let $u=0$. By the proof of Corollary 1 , the matrix $\mathbf{T}$ in Theorem 3 is the submatrix of $\mathbf{T}^{\prime}$ consisting of $8, \ldots, 17$ rows and $8, \ldots, 17$ columns. Thus,

$$
\mathbf{T}=\left[\begin{array}{llllllllll}
0 & 0 & 0 & 0 & 0 & 1 & 1 & 0 & 0 & 0 \\
0 & 0 & 0 & 0 & 0 & 0 & 0 & 0 & 1 & 1 \\
0 & 0 & 0 & 0 & 1 & 0 & 0 & 0 & 0 & 0 \\
0 & 0 & 0 & 0 & 0 & 0 & 0 & 1 & 0 & 0 \\
0 & 1 & 1 & 1 & 0 & 0 & 0 & 0 & 0 & 0 \\
1 & 1 & 0 & 0 & 0 & 0 & 0 & 0 & 0 & 0 \\
0 & 0 & 0 & 0 & 0 & 0 & 0 & 1 & 0 & 0 \\
1 & 0 & 1 & 1 & 0 & 0 & 0 & 0 & 0 & 0 \\
1 & 1 & 0 & 0 & 0 & 0 & 0 & 0 & 0 & 0 \\
0 & 0 & 0 & 0 & 1 & 0 & 0 & 0 & 0 & 0
\end{array}\right]
$$

By Theorem 3, we have

$$
\operatorname{det}\left(\mathbf{I}_{10}-t \mathbf{T}\right)=\zeta(H, t)^{-1}
$$

\section{Acknowledgment}

This research was partially supported by Grant-in-Aid for Science Research (C).

We would like to thank the referee for many valuable comments and many helpful suggestions. Also, we are indebted to the referee who gives us many remarks for our future study on zeta functions of hypergraphs.

\section{References}

[1] L. Bartholdi, Counting paths in graphs, Enseign. Math. 45 (1999), 83-131.

[2] H. Bass, The Ihara-Selberg zeta function of a tree lattice, Internat. J. Math. 3 (1992) 717-797.

[3] D. Foata and D. Zeilberger, A combinatorial proof of Bass's evaluations of the IharaSelberg zeta function for graphs, Trans. Amer. Math. Soc. 351 (1999), 2257-2274.

[4] K. Hashimoto, Zeta Functions of Finite Graphs and Representations of $p$-Adic Groups, Adv. Stud. Pure Math. Vol. 15, Academic Press, New York, 1989, pp. 211280.

[5] K. Hashimoto, Artin-type $L$-functions and the density theorem for prime cycles on finite graphs, Internat. J. Math. 3 (1992), 809-826.

[6] Y. Ihara, On discrete subgroups of the two by two projective linear group over $p$-adic fields, J. Math. Soc. Japan 18 (1966) 219-235.

[7] M. Kotani and T. Sunada, Zeta functions of finite graphs, J. Math. Sci. U. Tokyo 7 (2000) 7-25. 
[8] I. Sato, Bartholdi zeta functions for hypergraphs, Electronic J. Combin. 13 (2006).

[9] H. M. Stark and A. A. Terras, Zeta functions of finite graphs and coverings, Adv. Math. 121 (1996), 124-165.

[10] C. K. Storm, The zeta function of a hypergraph, Electronic J. Combin. 13 (2006).

[11] T. Sunada, L-Functions in Geometry and Some Applications, in Lecture Notes in Math., Vol. 1201, Springer-Verlag, New York, 1986, pp. 266-284.

[12] T. Sunada, Fundamental Groups and Laplacians (in Japanese), Kinokuniya, Tokyo, 1988. 